\title{
Spatial separation of left and right
} ventricular leads adjusted to the left ventricular end-diastolic dimension does not affect the change of the paced QRS complex duration in resynchronization therapy

\author{
Ewa Świerżyńska', Przemysław Mitkowski², Joanna Zakrzewska-Koperska1', \\ Artur Oręziak ${ }^{1}$, Rafał Baranowski', Maria Bilińska', Maciej Sterliński \\ 1 1st Department of Arrhythmia, National Institute of Cardiology, Warsaw, Poland \\ 2 1st Department of Cardiology, Poznan University of Medical Sciences, Poznań, Poland
}

Correspondence to: Ewa Świerżyńska, MSc 1st Department of Arrhythmia, National Institute of Cardiology, ul. Alpejska 42, 04-628 Warszawa, Poland, phone: +48223434619 , email: eswierzynska@ikard.pl Received: July 15, 2020. Revision accepted: August 31, 2020. Published online: September 8, 2020. Kardiol Pol. 2020; 78 (11): 1159-1161 doi:10.33963/KP.15595 Copyright by the Author(s), 2020
Introduction Cardiac resynchronization therapy (CRT) is a treatment option for patients with advanced heart failure, reduced left ventricular ejection fraction, and intraventricular conduction disturbances. ${ }^{1}$ Despite over 20 years of experience, indications for implantation, implantation techniques, and optimal device programming remain crucial research topics, because up to $30 \%$ of patients do not benefit from treatment. ${ }^{2,3}$ There are various indicators for determining CRT effectiveness and numerous predictors of treatment response have been identified, but some of them are of limited value. ${ }^{4}$ One of the secondary endpoints that aroused recent research interest is the reduction $(\Delta)$ of QRS complex duration as a result of dual-chamber capture. ${ }^{2,3,5,6}$ In this study, we aimed to analyze this issue based on the assumption that QRS complex shortening and the subsequent response achieved by CRT may depend on the location of right and left ventricular leads and the "separate leads as far as achievable" rule is still followed regardless of very limited and weak evidence. ${ }^{7-9}$

Methods No bioethics committee approval was required for this study. The project was carried out as part of a diploma thesis.

The study was a retrospective, single-center analysis of the relationship of spatial right ventricular (RV) and left ventricular (LV) lead arrangement and $\triangle Q R S: 1$ ) The spatial distance between RV and LV leads was analyzed based on routine chest X-ray images after implantation. Implantation procedures were not intended to maximize RV-LV electrode separation, and operators were not required to follow any specific protocol associated with this study. A $90^{\circ}$ angle between the lead tips was determined (including the selected stimulation configuration and the point on the LV multipole lead) using medical image viewer tools in the DICOM format; 2) On the chest $X$-ray image in the posterior-anterior projection, the length of the sides of that angle was determined, corresponding to $\mathrm{x}$ and y axes according to the Cartesian coordinate system. Similarly, chest X-ray was taken in the lateral projection where the horizontal distance ( $\mathrm{z}$ axis) corresponded with the $\mathrm{z}$ value; 3) The distance between the lead tips was calculated from the formula $\left.\sqrt{ }\left(x^{2}+y^{2}+z^{2}\right) ; 4\right)$ The time of ventricular muscle depolarization was calculated on a 12-lead electrocardiogram, on the precordial lead $\mathrm{V}_{6}$ before and after implantation of the CRT system. Atrio- and interventricular delays as well as other parameters of the CRT generator were set by the empiric mode and not modified for the purposes of the study. The mean values of the paced QRS measurement were as 

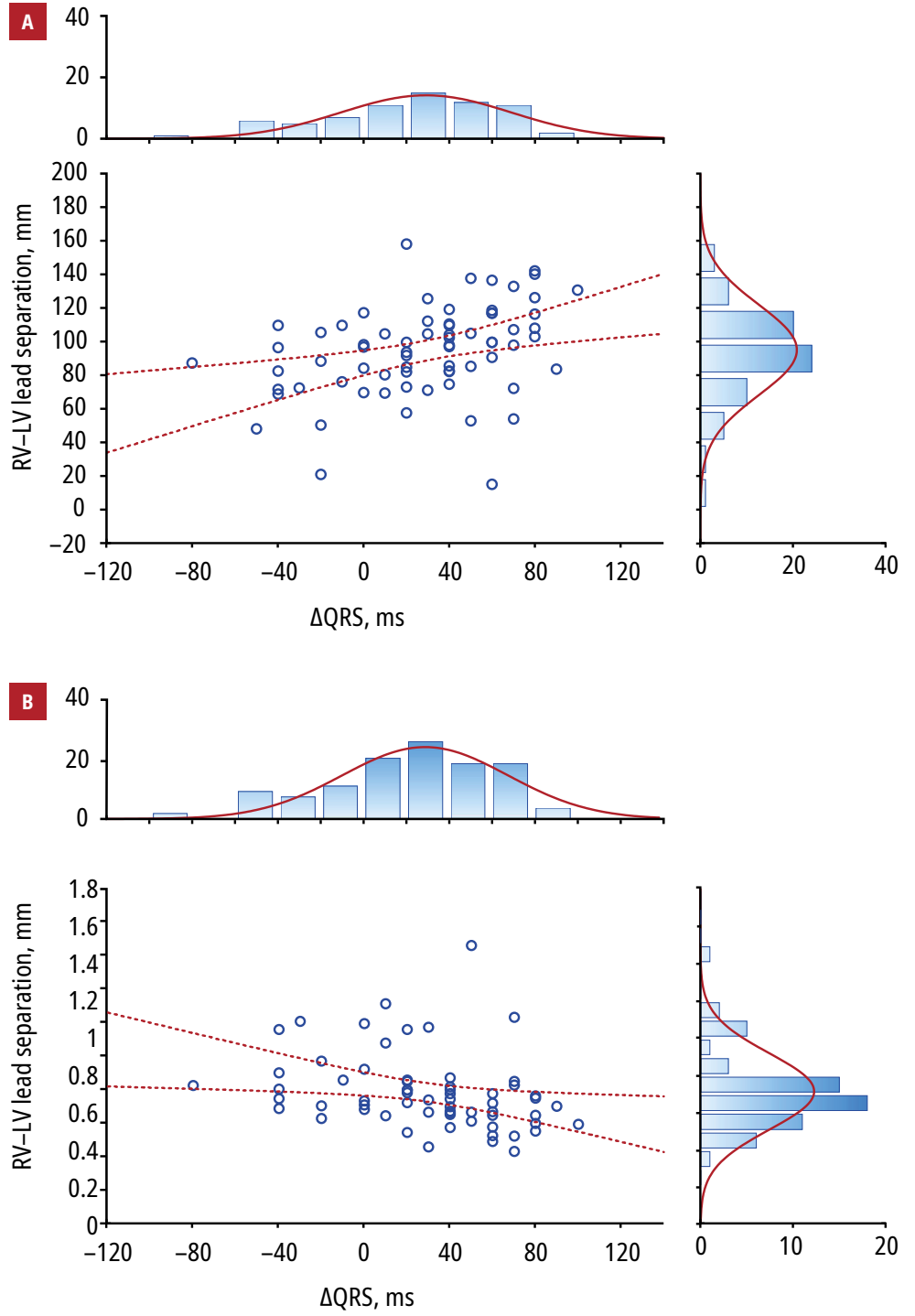

FIGURE 1 A - correlation between the spatial separation of right and left ventricular (RV-LV) leads and $\triangle Q R S$; B - correlation between the spatial separation of RV-LV leads adjusted to the left ventricular end-diastolic dimension and $\triangle Q R S$. The points on the graph correspond to each case. The density of cases in the area defined by the dashed lines reflects the strength of the correlation. Case distribution graphs are presented on the top and on the right, where the bars represent the number of cases with variable intervals (horizontal bars for $\triangle Q R S$ [ms] and vertical bars for RV-LV lead separation [mm]), and the solid line shows the Gaussian curve.

follows: atrioventricular delay, $155 \mathrm{~ms}$; interventricular delay, $10 \mathrm{~ms}$; 5) The correlation coefficient between lead separation and the difference in the duration of ventricular depolarization (where positive values of $\triangle \mathrm{QRS}$ mean QRS shortening) was calculated using the Pearson correlation test; 6) Measurements of electrode separation distances were standardized by left ventricular end-diastolic dimension (LVEDD) measurements on echocardiography and the correlation with the change in the duration of the QRS complex was recalculated.

Statistical analysis All calculations were performed using the Statistica software, version 13 (StatSoft, Tulsa, Oklahoma, United States).
The Shapiro-Wilk test was used to test the normality of data distribution. Qualitative variables (sex, occurrence of coronary artery disease, myocardial infarction, left bundle-branch block, New York Heart Association [NYHA] functional class) were expressed as percentages. Quantitative variables (age, left ventricular ejection fraction, QRS before CRT system implantation, QRS after CRT system implantation, distance between the left and right ventricular lead tips, LVEDD) were expressed as mean (SD) and minimum-maximum ranges. The Pearson correlation was used to assess relationships between lead separation and the difference in the duration of ventricular depolarization. A $P$ value less than 0.05 was considered significant.

Results and discussion The study group included 70 patients (men, $84.3 \%$ ) at a mean (SD) age of 73.8 (14.3) years. Up to 59\% of the study patients had coronary artery disease (myocardial infarction, $40 \%$ ), and $44 \%$ of them were in NYHA class III or IV and had heart failure with mean left ventricular ejection fraction of $28 \%$. A total of $67 \%$ of the patients had left bundle-branch block. Parameters expressing the impact of the spatial arrangement of ventricular leads on the change in QRS complex duration before CRT system implantation were as follows: mean (SD), 159 (33) ms; median, $160 \mathrm{~ms}$; minimum-maximum, 90-240 ms; and after CRT system implantation: mean (SD), 131 (28) ms; median, $120 \mathrm{~ms}$; minimum-maximum, 70-230 ms. The distance between left and right ventricular lead tips was: mean (SD), 94.47 (26.78) mm; median, $97.45 \mathrm{~mm}$; minimum-maximum, 15-158 $\mathrm{mm}$, and LVEDD was: mean (SD), 66.04 (10.66) mm; median, $65 \mathrm{~mm}$; minimum-maximum, 42-101 $\mathrm{mm}$.

The correlation between the right and left ventricular tip distance and $\triangle \mathrm{QRS}$ before and after implantation demonstrated power of $r=0.37(P=0.03)$. The correlation coefficient for the duration of the QRS complex before CRT system implantation and the distance between left and right ventricular lead tips was $r=0.34$ $(P=0.04)$. After standardizing the separation of leads by LVEDD, the distance correlation coefficient compared with $\triangle \mathrm{QRS}$ before and after implantation was nonsignificant $(r=-0.12$; $P=0.49$; FIGURE 1). The change in QRS complex duration after CRT system implantation depends on the location of the leads, the electromechanical properties of the myocardium, 5, ,6,10 and, as we have shown, only marginally on lead geometric distance. Considering the above conclusion and the fact that the distance between the leads is related to the size of the heart, it can be assumed that any difference in the QRS complex duration is correlated with heart size. After adjustment of the main result to LVEDD values, correlation coefficients for these parameters turned out to 
be nonsignificant, which seems to contradict the relationship between lead separation and the change of the QRS length. Increased distance between right and left ventricular leads has been shown to affect short- and mid-term CRT outcomes; however, the cited studies were relatively small and included endpoints such as LVEDD, NYHA class, or N-terminal fragment of the prohormone brain natriuretic peptide level reduction. ${ }^{5,7-9}$ We used the QRS complex as a factor that can be measured immediately post hoc, and its value as a predictor of CRT response has recently increased. ${ }^{6,11}$ Lead separation, which may drive QRS shortening, depends on numerous modifiable and nonmodifiable factors. ${ }^{12,13}$ However, in our view, it can be recommended as a method to enhance CRT response. One should also be aware of the fact that the direct pacing of the cardiac conduction system will be an alternative to or take an adjuvant position to CRT in the near future. ${ }^{14}$

Conclusions Geometrical ventricular lead separation for CRT affects the change in QRS complex duration. Although the distance between the tips of right ventricular and left ventricular leads is related to QRS shortening, this effect disappears after adjustment to LVEDD.

\section{ARTICLE INFORMATION}

CONFLICT OF INTEREST None declared.

OPEN ACCESS This is an Open Access article distributed under the terms of the Creative Commons Attribution-NonCommercial-NoDerivatives $4.0 \mathrm{In}$ ternational License (CC BY-NC-ND 4.0), allowing third parties to download articles and share them with others, provided the original work is properly cited, not changed in any way, distributed under the same license, and used for noncommercial purposes only. For commercial use, please contact the journal office at kardiologiapolska@ptkardio.pl.

HOW TO CITE Świerżyńska E, Mitkowski P, Zakrzewska-Koperska J, et al. Spatial separation of left and right ventricular leads adjusted to the left ventricular end-diastolic dimension does not affect the change of the paced QRS complex duration in resynchronization therapy. Kardiol Pol. 2020; 78: 1159-1161. doi:10.33963/KP.15595

\section{REFERENCES}

12013 ESC Guidelines on cardiac pacing and cardiac resynchronization therapy: the Task Force on cardiac pacing and resynchronization therapy of the European Society of Cardiology (ESC). Developed in collaboration with the European Heart Rhythm Association (EHRA). Eur Heart J. 2013; 34, 2281-2329.

2 Cleland JG, Abraham WT, Linde C, et al. An individual patient meta-analysis of five randomized trials assessing the effect of cardiac resynchronization therapy on morbidity and mortality in patient with symptomatic heart failure. Eur Heart J. 2013; 34: 3547-3556.

3 Butcher C, Mareev Y, Markides V, et al. Cardiac resynchronization therapy update: evolving indications, expanding benefit? Current Cardiology Reports. 2015; 17: 90.

4 Boidol J, Średniawa B, Kowalski 0, et al. Many response criteria are poor predictors of outcomes after cardiac resynchronization therapy: validation using data from the randomized trial. Europace. 2013; 15: 835-844.

5 Miranda RI, Nault M, Johri A, et al. Maximal electric separation-guided placement of right ventricular lead improves responders in cardiac resynchronization defibrillator therapy. Circ Arrhythm Electrophysiol. 2012; 5: 927-932.

6 Jastrzębski M, Baranchuk A, Fijorek K, et al. Cardiac resynchronization therapy-induced acute shortening of QRS duration predicts long-term mortality only in patients with left bundle branch block. Europace. 2019; 21:281-289.

7 Modi S, Yee R, Scholl D, et al. Ventricular pacing site separate on by cardiac computed tomography: validation for the prediction of clinical response to cardiac resynchronization therapy. Int J Cardiovasc Imaging. 2017; 33: 1433-1442.

8 Lang NN, Badar AA, Pettit SJ, et al. Interventricular lead separation is critical for NT-proBNP reduction after cardiac resynchronization therapy. Biomark Med. 2014; 8: 797-806.
9 Ariga R, Tayebjee MH, Benfield A, et al. Greater three-dimensional ventricular lead tip separation is associated with improved outcome after cardiac resynchronization therapy. Pacing Clin Electrophysiol. 2010; 33: 1490-1496.

10 Kydd AC, Khan FZ, Watson WD, et al. Prognostic benefit of optimum left ventricular lead position in cardiac resynchronization therapy: follow-up of the TARGET study cohort (Targeted Left Ventricular Lead Placement to guide Cardiac Resynchronization Therapy). JACC Heart Fail. 2014; 2: 205-212.

11 Kronborg MB, Nielsen JC, Mortensen PT. Electrocardiographic patterns and long-term clinical outcome in cardiac resynchronization therapy. Europace. 2010; 12: 216-222.

12 Kutyifa V, Kosztin A, Klein HU, et al. Left ventricular lead location and long-term outcomes in cardiac resynchronization therapy patients. JACC Clin Electrophysiol. 2018; 4: 1410-1420.

13 Bisch L, Da Costa A, Dauphinot V, et al. Predictive factors of difficult implantation procedure in cardiac resynchronization therapy. Europace. 2010; 12: 1141-1148.

14 Jastrzębski M, Moskal P, Bednarek A, et al. First Polish experience with permanent direct pacing of the left bundle branch. Kardiol Pol. 2019; 77: 580-581. 